\title{
La motivación y las matemáticas
}

\section{A motivação e as matemáticas}

\author{
Norfelino Pacheco-Carrascal ${ }^{1}$
}

Forma de citar: Pacheco-Carrascal, N. La motivación y las matemáticas. Revista Eco.Mat. 7 [149-158].

Recibido:

Abril 30 de 2015

Aceptado:

Agosto 4 de 2015
${ }^{1}$ Especialista en Orientacion Vocacional Profesional norfelino@gmail.com Institución Educativa Hermogenes Maza Cúcuta-Colombia

\section{Resumen}

Se presenta un breve punto de opinión con respecto a la importancia de la motivación, en el aprendizaje, en esta ocasión de las matemáticas, teniendo como punto de partida la falta de entusiasmo y deseo de aprender de los estudiantes con los que en el transcurso de mi labor académica y de la observación he visto evidenciada en los estudiante a los cuales durante varios años les he orientado esta asignatura, y que generalmente han mostrado poco interés por desarrollar su aprendizaje de manera al menos básica para salir adelante en sus cursos respectivos. Analizaremos, algunos aspectos como: ¿qué estar motivado?, ¿qué me motiva a realizar las cosas?, ¿de qué manera me han motivado a estudiar?¿cómo me han motivado a estudiar matemáticas?, a través de los cuales trataremos de entender y comprender como la motivación resulta ser un aspecto importante en el proceso de aprendizaje desde el punto de vista intrínseco y extrínseco en el estudiante. Además algunos aspectos de como motivar a nuestros estudiantes desde nuestra perspectiva de docentes.

Palabras clave: Aprendizaje de las matemáticas, metas y sueños, motivación, voluntad.

\begin{abstract}
This paper presents a brief point of opinion regarding the importance of motivation in the learning of mathematics, starting with the lack of enthusiasm and desire to learn that I have evidenced from students have oriented during my academic and observational work in this subject for several years, and have generally shown little interest in developing their learning in a least basic way to go forward in their respective courses.

We will analyze, some aspects like: What is being motivated? What motivates me to do things? How have I been motivated to study, how have I been motivated to study mathematics? Through these aspects we will try to understand how motivation turns out to be an important aspect in the learning process from the student's intrinsic and extrinsic standpoint. Also, some aspects of how to motivate our students from our teacher's perspective.
\end{abstract}

Keywords: Mathematics learning, goals and dreams, motivation, willing. 


\section{Resumo}

Apresenta-se um breve ponto de vista com respeito à importância da motivação na aprendizagem, neste caso, das matemáticas, tendo como ponto de partida a falta de entusiasmo e desejo de aprender dos estudantes, que no decorrer do meu trabalho académico e através da observação que tenho evidenciado como orientador durante vários anos de cursos de matemática, nos quais geralmente os alunos tem mostrado pouco interesse por desenvolver sua aprendizagem de maneira pelo menos básica para ter sucesso em seus cursos respectivos. Foram analisados alguns aspectos tais como: o que é estar motivado? ¿ o que me motiva a realizar as coisas? De que maneira me têm motivado a estudar? Como têm-me motivado a estudar matemáticas?. Através dos quais foi possível entender e compreender como a motivação resulta ser um aspecto importante no processo de aprendizagem desde o ponto de vista intrínseco e extrínseco no aluno. Além de alguns aspectos de como motivar aos estudantes desde nossa perspectiva como docentes.

Palavras-chave: Aprendizagem matemática, objetivos e sonhos, motivação, desejo.

\section{Introducción}

\section{1 ¿Qué es estar motivado? ¿ ¿Qué me motiva a realiza las cosas?}

Hoy en día varios autores han trabajado sobre la motivación desde diferentes roles y han dado a conocer sus teorías, como: la Teoría de necesidades de Maslow, la Teoría de expectativas de Victor Vroom, la Teoría de equidad de Stacey Adams, la Teoría de las necesidades de McClelland, entre otras, pero lo más importante, es saber que la motivación nos impulsa a realizar cosas, actividades y compromisos y por encima de cualquier obstáculo luchamos al máximo por conseguir nuestro propósito. La motivación es como un motorcito que nos impulsa, nos lleva y nos anima todo el tiempo a seguir la ruta trazada, con ahincó y coraje.

La motivación debe ser parte de nuestra vida, porque todo lo que queremos hacer necesita de impulso, de un deseo y esto es algo natural, por lo tanto la motivación nace con nosotros; porque si hacemos memoria, que nos impulsaría a caminar?, a hablar?, a decirle a mamá te quiero?, a decirle a papá quiero aprender montar bicicleta?

Todas estas cosas y muchas más de nuestra cotidianidad, tenían algo en común, y es ese agrado de alcanzar y disfrutar las cosas que añoramos, y para ello debemos estar totalmente motivados a realizarlas.

Así mismo McClellan dentro de su teoría, en uno de sus factores, nos enseña también que la motivación: "trata del impulso de obtener éxito y destacar. Y por tanto la motivación surge de establecer objetivos importantes, apuntando a la excelencia, con un enfoque en el trabajo bien realizado y la responsabilidad" McClellan (1961).

Estar motivado es estar lleno de expectativas en el desarrollo de nuestra personalidad, pues de esta manera luchamos por ser cada día mejor, en lo personal, social, laboral y en los diferentes aspectos de nuestra vida. 


\section{2 ¿De qué manera me han motivado a estudiar?}

Aquí encontramos dos alternativas:

Los que no fueron motivados por ninguna circunstancia, aquellos en los cuales la familia, el medio de vida, las amistades y su círculo social no fueron parte esencial e importante para tener la debida motivación de alcanzar quizás lo que algún día se soñó. Sin duda alguna y por más que queramos desmentirlo todos tuvimos sueños en nuestra infancia los cuales ante esta circunstancia quedan frustrados, pues no tuvo esa voz de aliento, esa voz de esperanza que le impulsara a conquistar esos ideales a pesar de las dificultades.

Por eso nuestros sueños como parte esencial de nuestra motivación intrínseca, son quienes nos animan a luchar por nuestra superación, es así que para (Ryan y Deici, 2000), la motivación intrínseca es aquella tendencia inherente a la búsqueda de la novedad y de los retos, a la extensión y ejercicios de las capacidades personales, a la exploración y el aprendizaje.

Acá también nos encontramos con muchos problemas sociales que nos afectan el deseo, la voluntad para poder superar los obstáculos; el empleo, las familias fraccionadas, la salud, las dificultades económicas, etc. Ante este panorama nos encontramos con aquellas personas que no tiene una situación estable en su modo de vivir, siendo este la causa de los problemas que hoy vemos y que día a día se multiplican más y más.

Por otro lado, Chiavenato (1999), define a la motivación como el resultado de la interacción entre el individuo y la situación que lo rodea. Dependiendo de la situación que viva el individuo en ese momento y de cómo la viva, habrá una interacción entre él y la situación que motivará o no al individuo.
Ante todo esto, las aspiraciones que se tienen son pocas; luego el estudiar, el prepararse académicamente, será algo sin importancia para un joven que vive ante este panorama. La motivación para estudiar es escasa ya que no encuentran una aplicación presente que le ayude a salir de sus dificultades, por lo tanto realizar otras actividades y que de alguna manera los ayude a salir de la dificultades sería su prioridad. Generalmente es salir a trabajar no importa la clase de trabajo lo importante es ganar para cubrir especialmente sus necesidades económicas presentes.

Pero es de notar también que algunos jóvenes a pesar sus circunstancias adversas han alcanzado sus logros, sus metas, con mucho esfuerzo y sacrificio. Hoy día trabajan satisfactoriamente $\mathrm{y}$ han mejorado su condición de vida y la de sus familias.

¿Qué los llevó a alcanzar sus metas?, su deseo de sobresalir, el deseo de destacarse en su contexto, el deseo de relacionarse profesionalmente con otros y sobre todo darle un giro a su entorno social.

Por tal razón, la motivación es un proceso que comienza con una necesidad insatisfecha, la cual crea una tendencia que estimula impulsos en el interior del individuo, logrando cambios en el comportamiento (Robbins 1994).

La otra alternativa es la de aquellos jóvenes que viven en un ambiente diferente, donde reina la armonía la paz y el ambiente es propicio para estar motivados a estudiar y a cumplir los sueños, poseen una gran autoestima que le ayuda a desarrollar sus potencialidades en busca de un horizonte mejor cada día, y todo gracias al gran ambiente familiar y la satisfacción económica que le permite acceder a muchas oportunidades de información y preparación personal, Jóvenes que crecen con ideales porque eso es lo que ven alrededor, por ejemplo en la mayoría de hogares y familias de estos jóvenes siempre están rodeados de
Enero-Diciembre 2016 ISSN 1794-8231 E-ISSN 2462 - 8794 PP: $149-158$ 
No. 1 alguna manera de las labores cotidianas de sus familiares y esta es una manera de formar y enseñar porque el ejemplo "ENSEÑA" alcanzando satisfactoriamente sus metas.

\section{3 ¿Cómo fue la motivación que me brindaron para querer estudiar matemáticas?}

En primer lugar para hablar de este aspecto, es importante reconocer que esta nace desde que nuestra conciencia empieza a dar luces de entendimiento y comprensión, y esto se da desde la edad comprendida entre los dos y siete años, etapa preoperacional (Piaget, 1968), desde allí aparecen las primeras estructuras intelectuales equilibradas, que permiten al niño la estructuración espacio-temporal y causal de acción práctica, porque empezamos a dar nuestros primeros pasos de aprendizaje, $\mathrm{y}$ todo marcha bien desde el momento en que nuestros padres nos dejan en la puerta de la escuela, hasta la llegada a nuestro hogar pasando por la hora final de actividades académicas, digo pasando porque hasta allí han pasado muchas cosas en nuestra vida, el trato con nuevos amiguitos, la aceptación o no de mis compañeritos o del maestro, el agrado por hacer una que otra actividad, el colorear, el hacer nuestras primeras caligrafías y el realizar nuestros primeros números, nuestros primeros cálculos, en general nuestro mundo de aprendizajes y todo lo vemos bello pero no se puede descifrar donde es el punto de quiebre, porque años más tarde esa ilusión que se observa inicialmente en el estudiante con el paso de los años desaparece. Esto se debe en gran parte a la falta de expectativas, tienden a formar aversión a las tareas, aburrimiento en la solución de problemas, en general se evidencia una falta de motivación la cual puede ser, intrínseca o extrínseca en la consecución de sus logros.

De allí que la motivación intrínseca se define como el de hacer una actividad para sus satisfacciones inherentes en lugar de alguna recompensa. Cuando una persona está intrínsecamente motivada actúa por diversión o por retos y no por presiones o recompensas externas. (Ryan y Deci, 2000).

En cuanto a la motivación extrínseca tiene también beneficios y perjuicios. Algunos de los beneficios de este tipo de motivación es que los estudiantes aprenden para obtener reconocimiento y mejores calificaciones, los estudiantes compiten en el aprendizaje por recompensas tangibles y además buscan logros más elevados.

Así como lo cita Tapia (2003) quien afirma que: "querer aprender y saber son las condiciones personales básicas que permiten la adquisición de nuevos conocimientos y la aplicación de lo aprendido de forma efectiva cuando se necesita". Según esta afirmación es necesaria la voluntad interés y deseo del estudiante para adquirir el aprendizaje, claro está teniendo en cuanta el papel importante del docente ya que mediante su buena disposición y actitud logran estimular también a sus "pupilos" y así mantener un ambiente de trabajo donde reine la armonía y la buena disposición y motivación en sus estudiantes.

Como se ha mencionado anteriormente, la motivación es como un motorcito que nos impulsa para hacer determinada tarea, acción. Este impulso o interés por desarrollar una actividad, se puede aplicar dentro del aprendizaje, lo cual resulta de gran ayuda para un buen desempeño académico de los estudiantes (Largo, Jaimes \& Largo, 2014).

González, Valle, Núñez y González (1996) establecen que la motivación es un proceso que abarca componentes muy diversos y que éstos componentes son aplicables de manera congruentes a la motivación académica.

Es así que para el aprendizaje: "La motivación académica es el término asociado con la motivación en el ámbito académico. Esta 
puede crear confianza en la capacidad, junto con un aumento del valor de la educación y el deseo de aprender". (Young, Johnson, Hawthorne y Pugh 2011).

Teniendo en cuenta lo anterior y las expectativas creadas en el aula de clase durante sus años de estudio y aprendizaje el estudiante empieza a disfrutar de unas asignaturas más que otras, de acuerdo a como se les han orientado estas.

Entonces aparece entre ellos una escala de valoración en las asignaturas, de acuerdo a sus gustos preferencias, aprendizaje, motivación e interés que despiertan ellas en el interior de cada estudiante.

Es muy frecuente que en esta escala de valoración los jóvenes no encuentran la Matemática como una de sus asignaturas predilectas, pues siempre se escucha en ellos y en el común de las personas que son aburridas, tediosas y que no llaman la atención el estudiarla y menos tratar de adquirir un aprendizaje de ellas. Ante este panorama es evidente la falta de interés y motivación entonces que despierta el estudio de estas.

Como explica (Miguez, 2005), la motivación está muy relacionada con el aprendizaje $y$ de alguna manera afecta positiva $o$ negativamente. La falta de motivación dentro del aprendizaje, es una de las principales causas de problemas en el aprendizaje. Los estudiantes motivados aprenden con mayor rapidez, y más eficazmente, que los estudiantes que no están motivados.

Ahora si el estudiante se fija claramente el propósito y determinación de alcanzar un aprendizaje efectivo es porque posee un comportamiento motivado, de seguro es gracias a el estímulo de sus familiares, de sus compañeros, profesores y del entorno donde se desenvuelven. Siendo este un estudiante que no tendría dentro de su perspectiva una necesidad aparente de someter su aprendizaje a una escala de valores.

Pues según Leavitt (1964) propone que la motivación es el comportamiento que está orientado hacia alguna meta y es causado por estímulos internos y externos

\section{Motivación en el Aprendizaje de las Matemáticas}

Estar motivado para aprender un determinado aspecto, laborar, social o académico siempre debe tener algunas características, de allí que para el aprendizaje de las matemáticas me he centrado en dos causas específicas; el hogar y la escuela, pues en estos dos conceptos se encierra la cotidianidad del estudiante en lo que hace referencia a su aprendizaje.

\subsection{Su aprendizaje en el Hogar}

No hay un correcto acompañamiento de los padres en el proceso de aprendizaje matemático (análisis, interpretación y solución de situaciones problemáticas), debido a que los padres seguramente no han tenido un buen desempeño en el estudio y aprendizaje esta área. Lo cual limita al estudiante desde su hogar a enriquecer su aprendizaje en este campo.

Caso contrario sucede en otras áreas, donde los padres retoman las tareas en las cuales hay que solo hacer resumen, o dibujar o pegar o consultar, o leer, o redactar y trascribir al cuaderno. Pero en matemáticas no. Es aquí donde en muchas ocasiones los mismos padres no dan al niño la motivación, ya que comentarios negativos no ayudan en este caso, por ejemplo padres que delante de sus hijos argumentan: "Es que yo también tuve problemas con la matemática y siempre se me dificultó, estos cometarios ya marca el pensamiento del niño", pues no olvidemos que somos seres humanos con sentimientos $\mathrm{y}$ emociones que pueden trascender para 
No. 1

Es así que las emociones no solo dependen de muchos factores personales (autoconcepto, creencias, actitudes, expectativas, etc.), sino también de factores contextuales como: la forma en que se le presente la situación de aprendizaje o lo atractiva e interesante que le resulte al estudiante (Bacete y Domench, 1997).

Ahora por otro lado tenemos entonces la otra cara de la moneda y es cuando los padres si hacen un acompañamiento preciso en el aprendizaje de las matemáticas desde sus conocimientos y aprendizaje adquiridos en su preparación personal, o en su defecto siempre buscan o encuentran a alguien para que acompañe a su hijo en sus actividades de aprendizaje matemático, lo cual traerá como resultado en la mayoría de los casos que los alumnos se motiven en su preparación, pues se sentirán seguros, sin temores y darán todo de sí para alcanzar sus logros.

\subsection{Su aprendizaje en la Escuela}

Y aquí hacen parte del proceso; las estrategias y metodologías impartidas por los docentes, donde en algún momento, la rutina se hace evidente y no hay un grado de alegría de motivación, que despierte el interés del estudiante, $\mathrm{y}$ tome aprecio por el desarrollo de sus actividades matemáticas, no importando si esta está mal, porque si está mal, se debe motivar el esfuerzo que ha hecho el estudiante, pues a su debido tiempo se puede desarrollar la actividad, corrigiendo los errores y asignando un nuevo compromiso y así evidenciar el deseo y motivación que tiene o no el estudiante por su aprendizaje.

March (2006) plantea que a lo largo del tiempo han surgido nuevos métodos de aprendizaje, los cuales se vinculan al cambio constante y lo que se busca finalmente es la adecuación de dichas teorías y métodos de aprendizaje a estos cambios. Las nuevas formas de aprender se desarrollan para lograr diversidad de objetivos en los estudiantes como, motivación, responsabilidad, capacidad de análisis, comunicación, entre otras.

Por otro lado si el estudiante no refleja ningún agrado por su aprendizaje matemático y su aplicación en diversos contextos y en el desarrollo de actividades que estimulen las competencias básicas matemáticas y su aplicación, que son el pilar del verdadero aprendizaje, esto se convierte en una gran dificultad para los años venideros, porque el alumno empezaría a mostrar el rechazo hacia el estudio de las matemáticas.

Esta es la actitud que no permite que el joven desarrolle sus potencialidades, ya que siente que no es capaz de realizar las actividades matemáticas porque en este momento su forma de pensar bloquea su capacidad de análisis y de resolución de problemas, y es por ello que vemos como se dejan llenar de tedio y se reprimen a mostrar sus habilidades por temor a no alcanzar lo que se proponen.

Lo indispensable entonces según Corno y Snow (1986), que los estudiantes pueden estar motivados por dos razones; la necesidad de alcanzar el éxito y la necesidad de evitar el fracaso que se combinan y producen una motivación individual para alcanzar o evadir el aprendizaje y resolver problemas que reten su habilidad o requieran mucho esfuerzo.

Por lo tanto es importante que siempre se evidencie la motivación por alcanzar los logros y esto consiste en "analizar como el individuo construye la situación, interpreta los eventos de dicha situación y procesa la información de esa situación". (Dweck, 1986).

Bello (1997), opina que la motivación "designa una construcción teórica para 
comprender las condiciones que activan una conducta y la dirigen hacia un fin u objetivo determinado". Otra definición importante la hace Romero (1985), el opina "la motivación se refiere, en general, a estados internos que energizan y dirigen la conducta hacia metas específicas"

Según esto entonces es importante saber que el estudio de la matemática necesita urgentemente, la compañía de padres, docentes e instituciones que le apunten al desarrollo motivacional en todos sus aspectos en la adquisición de conocimientos, realización de actividades y análisis de procesos matemáticos con nuevas estrategias y metodologías, pero ante todo una actitud diferente que llene de expectativas, sueños e ilusiones a quienes inician en sus labores académicas el recorrido por los caminos de las matemáticas.

Por otro lado todos los actores que se encuentran en el entorno, donde el estudiante se desenvuelve, deben expresar confianza, cariño y respeto, para llenar de motivación a ese ser que llega al encuentro con el mundo de las matemáticas. Y así vea con gran satisfacción el estudio de esta y le permita el desarrollo de sus potencialidades y habilidades matemáticas inherentes en él.

Con respecto a esto Tapia (2003) dice que las metas constituyen la principal variable que influye en la motivación y establece cuatro tipos de metas: las relacionadas con la tarea, las relacionadas con la autovaloración, las relacionadas con la valoración social y las relacionadas con la consecución de recompensas externas.

Según lo anterior se puede decir que la primera meta se puede lograr en el momento que el estudiante tome la iniciativa $y$ voluntad de dedicar el tiempo y esfuerzo necesario para su aprendizaje matemático, mediante el cumplimiento de las diferentes actividades enfocadas a un desarrollo de habilidades en la solución de problemas cotidianos, dando rienda suelta a su aprendizaje. La segunda meta se logra cuando el estudiante al reconocer su gran dedicación en el estudio y enriquecimiento matemático, valora su esfuerzo y sacrificio por alcanzar sus ideales específicamente cuando logra superar sus dificultades académicas, para alcanzar sus objetivos más cercanos con lo cual va construyendo y armonizando su nivel de comprensión y análisis matemático. La tercera cuando empieza a valorar su entorno y la necesidad de empezar a dar frutos por mejorar su condición de vida de la cual hacen parte un sin número de personas que lo rodean y siempre esperaran lo mejor de él. Y la última meta cuando ve realizado sus sueños a través de los estímulos y recompensas por su gran esfuerzo, que con el tiempo van llegando y acrecentando su espíritu de superación y valor hacia lo que realiza.

Para Zemelman (1998), el objetivo principal al enseñar matemáticas es ayudar a que todos los estudiantes desarrollen capacidad matemática. Los estudiantes deben desarrollar la comprensión de los conceptos y procedimientos matemáticos y deben estar en capacidad de ver y creer que las matemáticas hacen sentido y que son útiles para ellos. Maestros y estudiantes deben reconocer que la habilidad matemática es parte normal de la habilidad mental de todas las personas, no solamente de unos pocos dotados.

Otro investigador que escribe sobre esto es Ángulo (2006), quien opina que enseñar matemáticas es proporcionar medios de reflexión para evaluar y disciplinar estructuras cognoscitivas compatibles con un marco referencial de orden platónico. Este mismo autor agrega que la matemática por ser una ciencia antigua ha tenido que ir cambiando $y$ adaptándose a los cambios que ocurren a lo largo del tiempo, ya que al transcurrir estos cambios, los seres humanos buscan la mejora
Enero-Diciembre 2016 ISSN 1794-8231 E-ISSN 2462 - 8794 PP: $149-158$

\section{5}


No. 1 en su sobrevivencia y la matemática brinda la oportunidad de modificar o crear una mejora en su contorno.

Hoy en día los estudiantes que se encuentran en los salones de clases son estudiantes nacidos en era de la tecnología y los profesores se tienen que integrar a esta nueva onda. Es por ello necesario crear actividades donde se puedan utilizar estos medios y brindarle a los estudiantes estimulaciones donde ellos se sientan cómodos y donde puedan manejar sus conocimientos de una manera adecuada, siempre con el profesor como guía para lograr estos objetivos.

(Farías y Pérez, 2010).

\section{Estrategias motivadoras}

Ante todo este panorama que nos relaciona el aprendizaje matemático - estudiante motivación, nos queda por dar una mirada a las alternativas que posee el docente como estrategias que motiven el aprendizaje matemático.

Es así como dentro del aprendizaje, existen diversos métodos para aprender que buscan que el estudiante siempre este motivado a estudiar, que de una forma u otra le resulte fácil o divertido hacerlo y que además de los conocimientos, obtenga otros beneficios como investigar, participar, descubrir, aportar, tolerar, relacionarse con otros entre otros.

Una alternativa es el aprendizaje cooperativo, permitir el trabajo de aula en pequeños grupos y su evaluación se da según el resultado del trabajo de todos. Es útil porque ayuda a desarrollar competencias y beneficia las habilidades interpersonales.(March 2006).

Como lo confirma Johnson, Johnson y Holubec, (1999) "Aprendizaje cooperativo es el empleo didáctico de grupos reducidos en los que los que los alumnos trabajan juntos para maximizar su propio aprendizaje y el de los demás”.
El juego es otra alternativa que nos facilitaría en gran medida nuestro deseo de motivar nuestros estudiantes, de esta manera se sienten estimulados, ya que es una forma de aprendizaje que motiva y desarrolla competencias.

Malone y Lepper (1987), explican que el aprendizaje puede convertirse en algo divertido, que genere motivación a los estudiantes. Plantea que cuando una persona esta "retado" a alcanzar una meta en particular, puede llegar a mostrar más interés frente a esa actividad.

La simulación hace parte también de las opciones que podemos utilizar para motivar nuestro quehacer pedagógico, ya que en esta los estudiantes pueden jugar una estrategia en particular o pueden adoptar también un enfoque determinado. Si el estudiante falla o no alcanza el resultado que desea, puede volver a intentarlo con una perspectiva diferente, una estrategia modificada o con algo nuevo para implementar.

Según Corti (2006), en la simulación se mide también la capacidad de evaluar, qué estrategias de aprendizaje aprobó, lo bien que analizó y dio sentido a la información, lo bien que explica y justifica sus decisiones y lo que bien que entendía de cómo la decisión afectas un resultado especifico.

En general, cuando los alumnos, el profesor y el contenido de las materias (matemática) son congruentes y se muestran interesantes, la educación va a ser más amena para ambas partes, pues según Hernández (2002), así se hallara satisfacción en el estudio. El profesor podrá enseñar el contenido de la materia y estimular la motivación. Los estudiantes podrán adquirir conocimientos y mostrarse motivados, pues tendrán dominio de la materia, buenos resultados y reconocimiento. 
De todo lo anteriormente expuesto, es fácil concluir que todos estamos perfectamente equipados para aprender en nuestra vida un gran sin número de eventos que día a día nos hace competentes en todas nuestras actividades y las matemáticas no deben ser la excepción, pues nuestra mente unida a nuestra voluntad y motivación de alcanzar nuestros ideales y metas es un gran instrumento capaz de lograr lo que nos proponemos. Emulando una frase muy motivadora de una película de dibujos animados que afirmaba "Cualquiera puede cocinar", yo también podría decir que:

"Cualquiera con un espíritu de aventurero lleno de motivación y deseo por alcanzar sueños puede aprender matemáticas"

\section{Referencias}

Bacete, G., \& Doménech, F. (1997). Motivación, aprendizaje y rendimiento escolar. Revista de motivación y emoción. Universidad Jaume I de Castellón, 1(6), 2435.

Bello, P. J. (1997). Motivación en tu vida. Venezuela: Editorial Panapo.

Corno, L. \& Snow, R.E. (1986): Adapting teaching to individual differences among learners. En M. Wittrock (Ed.): Handbook of research on teaching. New York: McMillan

Corti, K. (2006). Games-based Learning; a serious business application. PIXELearning, $1-20$.

Chiavenato, I. (1999). Administración de Recursos Humanos. Colombia: Editorial Mc Graw Hill

Dweck, C.S. \& Elliot, E. S. (1986). Achievement motivation. En E.M. Hetherington (ed.) Socialization, personality and social development. Wiley y Sons, Nueva. York-USA.
Farías D. \& Pérez J. (2010). Motivación en la Enseñanza de las Matemáticas y la Administración. Formación Universitaria, 3(6), 33-40.

Gonzalez, R., Valle, A., Nuñez, J., \& Gonzalez, J. (1996). Una Aproximación Teórica al Concepto de Metas Académicas y su Relación con la Motivación Escolar. Psicothema, 8(1), 45-61.

Hernández, P. (2002). Psicología de la educación: Corrientes actuales y teorías aplicadas. México D.F: Trillas S.A.

Johnson, D., Johnson, R., \& Holubec, E. (1999). El aprendizaje cooperativo en el aula. Buenos Aires: Paidós.

Largo, M., Jaimes, P., \& Largo, Y. (2014). Abordando el aprendizaje de las matemáticas. Revista Ecomatemático, 5(1).60-65

Leavitt, H. (1964). Managerial psychology. The University of Chicago Press, Chicago and London: University of Chicago

Malone, T., \& Lepper, M. (1987). Making Learning Fun: A Taxonomy of Intrinsic Motivations for Learning. Lawrence Erlbaum Associates, 3(3), 223-253.

March, A. (2006). Metodologías activas para la formación de competencias. Educatio siglo $X X I, 24,35-56$.

McClelland, D. (1961). Teoría de las necesidades ISSN 1794-8231 E-ISSN $2462-8794$ PP: $149-158$

Miguez, M. (2005). El núcleo de una estrategia didáctica universitaria: motivación y compresión. Revista ieRed: Revista Electrónica de la Red de Investigación Educativa, 1(3), 1-11.

Piaget, J., (1968). Etapas del desarrollo cognitivo infantil. 
Ryan, R., \& Deci, E. (2000). Intrinsic and Extrinsic Motivations: Classic Definitions and New Directions. University of Rochester, Contemporary Educational Psychology, 25, 54-67.

Robbins, S., \& Judge, T. (1994). Comportamiento organizacional. Naucalpan de Juárez, Estado de México: Pearson Educacion.

Tapia, J. A., (2003). Motivar para Aprender. En: Herramientas para la Reflexión Pedagógica. Bogotá

Young A, J., Hawthorne M, \& Pugh J. (2011). Cultural predictors of academic motivation and achievement: a self-deterministic approach. Callege Student Journal, 45(1), 151-163.

Zemelman, S., Harvey, D. \& Hyde A. (1998). Best Practice: New Standards for Teaching and Learning in America's Schools, $2^{\text {a }}$ ed., United States of America: Editorial Hinemann. 